\title{
Cancer vaccines: progress reveals new complexities
}

\author{
Zhiya Yu and Nicholas P. Restifo \\ National Cancer Institute, National Institutes of Health, Bethesda, Maryland, USA \\ Address correspondence to: Nicholas P. Restifo, National Cancer Institute, Building 10, Room 2B/42, National Institutes of Health, \\ Bethesda, Maryland 20892-1502, USA. Phone: (301) 496-4904; Fax: (301) 402-0922; E-mail: restifo@nih.gov. \\ J. Clin. Invest. 110:289-294 (2002). doi:10.1172/JCI200216216.
}

A decade ago, it seemed clear that our burgeoning knowledge of the molecular identities of tumor-associated antigens and a deeper understanding of basic immunology would point the way to an effective therapeutic cancer vaccine. Significant progress has been made and objective regressions after immune-based treatments are observed in some patients - even in those with bulky, metastatic disease. Notwithstanding this progress, we do not yet have a cancer vaccine in hand that can reliably increase patient survival or induce tumor destruction.

The creation of therapeutic cancer vaccines has proven to be an enormous challenge, and many of the strategies learned in the development of highly successful vaccines against infectious agents simply do not apply to cancer vaccines. Issues of antigenic change and immune escape are present in both antitumor and antiviral situations. However, one big difference between antiviral and antitumor vaccines is that the former are preventative whereas the latter are generally expected to be therapeutic.

Another problem with the targeting of tumor antigens relates to their poor immunogenicity. Tumor antigens appear to be relatively well tolerated in the host, perhaps because many of these antigens are also expressed in normal tissues. In this context, a successful cancer vaccine raises the specter of autoimmune attack if the vaccines are ultimately powerful enough to eliminate cancer cells. In this review, we highlight new challenges that have been revealed by recent progress in the field of tumor immunology. We then attempt to outline a future plan for cancer immunotherapy.

\section{The existence of tumor-specific immune responses in cancer patients}

The first reports that immune responses might result in tumor regression came over a century ago from William Coley, who treated cancer patients with live bacterial cultures, nonspecifically activating their immune systems. In the 1980s, Rosenberg et al. pioneered the use of high doses of the $T$ cell growth factor IL-2 in individuals with metastatic kidney cancer or melanoma and achieved objective cancer regressions in $15-20 \%$ of treated patients (1). Because IL-2 is not known to have direct effects on the growth of solid tumors, its antitumor activities are most likely associ- ated with its ability to expand lymphocytes, including the low-affinity $T$ cells.

The most striking evidence for naturally occurring antitumor immune responses comes from rare anecdotal observations of spontaneous regressions of tumors in patients with cancer. Also rare, but somewhat more amenable to study, is the observation of paraneoplastic autoimmunity that can accompany often occult malignancies. For example, high titers of serum IgG specific for immunogenic proteins expressed in both carcinomas and normal tissues have been detected in cancer patients with paraneoplastic neurological diseases (2). Because these antigens are generally found in the nucleus and cytoplasm, it is unlikely that the autoantibodies have direct effects on either tumors or normal tissues. However, they are proposed to be surrogate markers for activated cellular immune responses. Indeed, cytotoxic T lymphocytes specific for cdr-2 have been detected and linked to cerebellar degeneration in those seropositive patients with breast and ovarian cancers (2).

Autoimmunity has been modeled to a limited extent in animals, where vitiligo, the patchy and permanent depigmentation that results from the destruction of dermal melanocytes, has been found to accompany the regression of the experimental B16 melanoma in C57BL/6 mice. Vitiligo is also positively correlated with a favorable response to IL-2 in patients with metastatic melanoma $(3,4)$.

\section{Identification of tumor-associated antigens suitable for therapeutic targeting}

A variety of techniques have been applied to identify tumor antigens recognizable by tumor-specific $\mathrm{T}$ cells. None has been more successful than an approach that uses transient transfection of pools of genes from a tumor-derived cDNA library to confer recognition upon a target cell, thus identifying the gene encoding the target epitope. While cloning efforts have been prodigiously successful, protein chemists have also made inroads into the identification of target antigens by pushing the limits of high-performance liquid chromatography and tandem mass spectrometry. Peptides can now be eluted from MHC complexes derived from tumor cell membranes and characterized directly (5). In addition, it is possible to test candidate tumor antigens by the so- 
called reverse immunology method, specifically by sensitizing immune cells with the candidate antigen, then testing the ability of sensitized cells to specifically kill tumor cells that are known to express the antigen.

To date, approximately $70 \mathrm{MHC}$ class I- and class II-associated tumor antigens have been discovered, while more than 1,700 have been identified by antibodies in cancer patients. About ten antigens are currently known to be recognizable by both $\mathrm{T}$ cells and antibodies, although the actual number of antigens for which IgG production requires Th cells is probably much greater.

We do not know for certain how many of the candidate tumor antigens are suitable targets for tumor immunotherapy. A "valid" target antigen must be expressed specifically in the tumor, or at least be expressed at levels sufficiently higher there than in vital organs. For T cell-based therapy, it must be processed and presented in the context of MHC molecules. The need for positive and negative control tumor lines is often overlooked; rigorous (and numerous) controls are needed to convincingly demonstrate that a candidate antigen is a suitable target for use in an immunotherapy trial.

It has been estimated that $10^{5}$ to $10^{6} \mathrm{MHC} /$ peptide complexes are present on the surface of a typical cell (6), although some antigen-presenting cells may express more. Considering the redundancy of individual complexes in a given cell and the poor MHC expression and antigen processing in the majority of tumor cells, a maximum of approximately 10,000 different $\mathrm{MHC} /$ peptide complexes is likely to be presented on a tumor cell. With the assumption that dozens of peptide epitopes could be derived from an expressed protein, and additional antigens could be generated from alternative open reading frames, a conservative estimate of the total number of peptides that are actually able to bind any given MHC may exceed 1 million. Therefore, the chances that any given peptide will be presented on the tumor cell surface are approximately $1: 100$, which leaves $99 \%$ of the potentially recognizable $\mathrm{T}$ cell epitopes simply absent from the surface of a given target tumor cell. Therefore, it is clearly not valid to assume that expression of a mutated candidate antigen will result in the MHC-restricted presentation of that antigen on the tumor cell surface.

Despite practical and theoretical concerns about some putative tumor-associated antigens, there is already a large and growing list of antigens that have been convincingly shown to be valid targets for immunotherapy. The success of tumor antigen identification approaches put to rest the notion that spontaneous human tumors - unlike their experimental mouse counterparts - simply lacked the expression of antigens recognizable by the immune system.

\section{Enhancing tumor antigen immunogenicity by modifying epitope sequences}

Tumor antigens in their original form generally bind poorly to their restricting MHC molecules. In addition, peripheral autoreactive $\mathrm{T}$ cell precursors recognize their cognate peptide/MHC complexes with low affinity. Thus, most tumor antigens identified so far are poorly immunogenic in vivo. Dramatic increases in the magnitude of $\mathrm{T}$ cell responses and sensitivity to antigen stimulation have been observed in both human and mouse models using agonistic altered peptide ligands. These altered peptides are capable of enhancing the stability of peptide/MHC complexes because of modifications in the MHC anchor residues (7) or as the result of favorable and generally conservative changes to the peptide at the interface with the $T$ cell receptor (TCR) $(8,9)$. Most importantly, the enhanced T cell responses can retain their specificity to the native antigen, which allows them to kill target tumor cells ex vivo and, presumably, in vivo.

Both the association and the dissociation rates contribute to the steady-state stability of the interactions between peptide/MHC and ultimately TCR/peptide/MHC complexes. However, we have found that the enhancement of immunogenicity might be more affected by the off-rate than by the on-rate. For example, gp $100_{209(2 \mathrm{M})}$, an anchor-fixing modification of a native antigenic peptide derived from human melanoma antigen, gp $100_{209-217}$, is 100 -fold more potent in activation of naive $T$ cells than is wild-type peptide. The steady-state binding affinity of the modified peptide is nine times higher than that of the wildtype peptide. In contrast, the dissociation rate of modified peptide from HLA-A2 molecule is more than 100 -fold slower than that measured with the wild-type peptide (our unpublished data).

The stability of the target peptide/MHC complex is not only important in order to achieve the required antigenic density for naive $\mathrm{T}$ cell activation but may also alter the quality of the $T$ cell response. A study by Mark Davis's group concluded that a TCR bound to antagonist ligands with lower affinities because of an increased off-rate (10). Conversely, extending the offrate by amino acid substitutions may augment $\mathrm{T}$ cell "affinity maturation." Immunizing mice with agonistic peptide ligands elicits high-avidity $T$ cells that can recognize the relevant tumor cells, and even target cells pulsed at relatively low concentrations with weakly binding peptides (8). Studies from our laboratory have shown that although the human (hgp $100_{25-33}$ ) and mouse ( $\mathrm{mgp} 100_{25-33}$ ) epitopes are homologous, differences in the three $\mathrm{NH}_{2}$-terminal amino acids result in a $2-\log$ increase in the ability of the human peptide to stabilize "empty" $\mathrm{D}^{\mathrm{b}}$ MHC molecules and a 3-log increase in its ability to trigger IFN- $\gamma$ release by T cells (11). In a clinical trial, using anchor-modified gp100 peptide immunization in melanoma patients resulted in dramatic increase of tumor-reactive $T$ cell responses (12). Thus, modified antigenic peptide based on these ideas could be of significant value in vaccination against tolerant or weakly immunogenic tumor-associated cells.

\section{The nature of antitumor effector cells}

More than 40 years ago, Prehn and Main obtained evidence of specificity in the immune response to tumors. 
Mice immunized with irradiated methylcholanthreneinduced sarcoma cells, they showed, are fully protected against a subsequent challenge with that same tumor, but not with other tumors. Humoral responses against solid tumors may play some role in the effective killing of tumor targets either by augmenting antigen presentation or by the ligation of a growth factor receptor on cancer cells, such as HER-2/Neu. A large body of work using antibody depletion and gene knockout mice has revealed that both $\mathrm{CD}^{+}$and $\mathrm{CD} 4^{+} \mathrm{T}$ lymphocytes are crucial for therapeutic antitumor immune responses.

Compared with the comprehensive studies using $\mathrm{CD}^{+} \mathrm{T}$ cells in tumor models, relatively little is known about how $\mathrm{CD}^{+} \mathrm{T}$ cells influence antitumor immunity. Very early work demonstrated that disseminated murine leukemia could be eradicated by a combination of cyclophosphamide and adoptively transferred cells, now known to be $\mathrm{CD}^{+} \mathrm{T}$ cells (13). The most dramatic examples of the power of $\mathrm{CD}^{+} \mathrm{T}$ cells in the immune response to self-proteins can be found in murine models of autoimmune diseases, such as experimental allergic encephalomyelitis, systemic lupus erythematosus, and diabetes. In these models, disease can often be transferred to naive mice with purified, self-reactive $\mathrm{CD}^{+}$splenocytes or specific $\mathrm{CD}^{+} \mathrm{T}$ lymphocyte clones. Antigen-specific $\mathrm{CD} 4^{+} \mathrm{T}$ lymphocyte clones can also treat tumor through $\mathrm{CD}^{+} \mathrm{T}$ cells specific for the cognate antigen (14). Tumor antigen-specific $\mathrm{CD}^{+} \mathrm{T}$ cells have been isolated from tumor-infiltrating lymphocytes from melanoma patients (15). Adoptive transfer into patients of unfractionated tumor-specific $T$ cells has been shown to promote tumor regression (16). These studies and others suggest that the full activation of autoreactive $\mathrm{CD} 4^{+} \mathrm{T}$ cells may be an important immune component that is currently missing from many current clinical cancer vaccine trials.

Natural killer cells kill many tumor cell lines and may also play a critical role in antitumor immunity. A recent study in transgenic mice lacking $\mathrm{NK} 1.1^{+} \mathrm{CD}^{-}$cells linked an impaired acute tumor rejection to deficiency of NK activity (17). NKG2D receptors expressed by NK cells and activated $\mathrm{CD} 8^{+} \mathrm{T}$ cells and macrophages can be stimulated by their ligands, which are often overexpressed on cancer cell lines (18). Most surprisingly, clinical results from hematopoietic cell transplantation revealed that alloreactive NK cells in the donor graft prevented leukemia relapse in leukemic recipients (19). Although the innate immune system is often ignored as an important component of the antitumor immune response, lessons learned about immunity to infectious microorganisms indicate that early host defenses help determine the nature of downstream adaptive immune responses. One recent line of investigation pursued in our laboratory concerns the initiation of apoptotic death that results in the engagement of key innate immune pathways; the consequences of cell death may induce dendritic cell activation and benefit immune induction (ref. 20; see also Steinman and Pope, this Perspective series, ref. 21). A new generation of nucleic acid vaccines encoding an alphaviral replicase enzyme, together with tumor antigens, induces apoptotic death coupled with antigen production. This approach leads to quantitative and qualitative enhancement of the therapeutic antitumor immune response in animal models $(22,23)$.

\section{Unexpected obstacles in early clinical trials}

With antigens in hand, immunotherapists set off to create a new class of therapeutic vaccines based on defined antigens. Recombinant immunogens were created using the same viruses that have proven themselves to be so successful in the realm of infectious diseases - including vaccinia, polio, and influenza $\mathrm{A}$ - as well as some others including adenoviruses and bird poxviruses (canarypox and fowlpox) $(24,25)$. In animal models, these vaccines can prime $\mathrm{T}$ cell responses and elicit powerful immune responses that lead to tumor cell destruction. However, when these viruses were tried in the clinic, it became apparent that experiments in animal models had failed to predict key aspects of recombinant vaccine function in people.

One reason for these disappointing results was preexisting neutralizing antibody. In one recent study in which patients were immunized with recombinant adenoviruses encoding the melanoma-associated antigens gp100 or MART-1, only 6 of 54 patients had neutralizing antibody titers of less than 100 , with the majority having neutralizing titers of more than 400 (26). Similar problems might be observed using clinical-grade recombinant vaccinia viruses. Patients thus retain strong anti-vaccinia antibodies for many decades after immunization - a lasting legacy of the worldwide immunization program to eradicate smallpox. One would fully expect pre-existing immunity to be a problem in the use of several other vectors under consideration, including recombinant versions of polio and transfectant influenza A viruses of the more commonly observed strains. One way to circumvent this problem would be to use viruses whose natural hosts are non-mammalian, such as the avian poxviruses (Letvin, this Perspective series, ref. 27). These viruses are antigenically distinct from poxviruses and are incapable of replicating in mammalian cells. Strategies employing genetically engineered influenza viruses may prove useful in the development of live virus vaccines against cancer (24).

Another potential obstacle uncovered in early clinical trials is immunity to an antigenically complex vaccine that is immunodominant over a response to a transgene-encoded weak tumor antigen. This problem, which was not adequately studied in early experimental animal models and remains exceedingly difficult to model in ongoing preclinical work, may be summarized as follows: Vectors may interfere with the induction of reactivity to the encoded tumor antigen through the poorly understood mechanisms of immunodominance. $T$ cell responses elicited by protein immunization tend to focus on one or a few sites in the antigen. Whether this phenomenon is driven by the predetermined TCR repertoire, the competition among T cells based on their affinity to antigenic determinants, or the characteristics of antigen processing is not 
known $(28,29)$. In viral vector-based vaccines, self-antigens are coexpressed with viral proteins. If the immunodominant sites reside in the viral components, the vaccine will fail to elicit the desired immune responses. The use of vaccines based on "naked" plasmid DNA vaccines (i.e., DNA without associated protein) may circumvent both pre-existing immunity and immunodominance. Despite these advantages, our own clinical work has shown no evidence of immunization or antitumor effect of naked DNA immunization against the gp100 tumor antigen (unpublished data), although naked DNA is effective in many animal models.

\section{Consistent increases in tumor-specific T cells without consistent clinical responses}

There is now incontrovertible evidence that precursor frequencies of tumor-specific $\mathrm{T}$ cells can be increased after immunization using several different tumor-associated antigens - including those antigens that are nonmutated "self" tissue differentiation antigens (12). The presence of increased antitumor $\mathrm{T}$ cell precursors after vaccination has been convincingly demonstrated in both mice and humans, using tetramer or ELISPOT analysis, real-time RT-PCR, and other techniques.

Thus far, the most effective immunization strategy in our patients with advanced melanoma has been vaccination with peptide emulsified in incomplete Freund's adjuvant. Immunization with a gp100-derived peptide modified to enhance its binding to HLA-A2 dramatically increased levels of peptide-specific $\mathrm{CD}^{+} \mathrm{T}$ cells in the peripheral blood. Importantly, these $\mathrm{T}$ cells recognized and killed a variety of melanoma cells that expressed the gp100 melanoma antigen and the restriction element HLA-A*0201 after culture ex vivo. Administration of IL-2 following peptide immunization resulted in significantly more objective tumor regressions than seen after IL-2 treatment alone (12). However, most of these responses turned out to be partial and transient, and most responding patients eventually succumbed to progressively growing tumor.

\section{Proposed mechanisms of tumor escape}

The current notion that tumor cells must "escape" immune recognition is based largely on the idea that neoantigens expressed by tumor cells as a consequence of their genetic instability will be immunogenic. There is little doubt that the tumor contains a large number of mutations (30) that can potentially generate new antigens recognizable by the immune system, but there is considerable doubt about what the immunological response to these potential immunogens will be. A number of groups have conducted experiments in which highly immunogenic foreign antigens, such as the hemagglutinin protein from influenza (31), the $\beta$-galactosidase enzyme from Escherichia coli (32), and the ovalbumin protein from the chicken, are expressed in tumor cells (33). The results are fairly uniform: tumors tend to grow progressively, retaining their lethality despite the expression of a foreign and highly immunogenic protein by the tumor cell.
Proposed mechanisms for tumor escape include those relating to the inherent genetic instability of tumor cells (34-38) and others that might be shared by many normal cells in the body. The latter include the lack of expression of costimulatory molecules (B7-1/CD80, B7-2/CD86, and CD40 ligand), the induction of suppressor cell activity, and the production of immunoinhibitory substances such as TGF- $\beta$ or IL-10. Many of these theories are intuitively appealing but lack direct experimental evidence or consistent results. For example, Fas ligand (FasL) has been proposed as a mediator of the tumor "counterattack." However, controlled experiments show that FasL expressed in animal tumor models results not in escape, but in more rapid rejection (39-42).

Several groups have proposed the loss of $\beta_{2}$-microglobulin $\left(\beta_{2} \mathrm{~m}\right)$ as a mechanism of immune escape. However, work by Karre, Snook, and colleagues in animal models demonstrated that the loss of $\beta_{2} \mathrm{~m}$, an essential and invariant subunit of class I MHC complexes, results in exquisite sensitivity to NK cell-mediated killing and leads to tumor elimination, not escape $(43,44)$. Although some human melanoma cells have also been shown to lose $\beta_{2} \mathrm{~m}$ with clinical progression, human $\beta_{2}$ m-deficient cells are also susceptible to NK cell-mediated killing (45). This evidence need not indicate that $\beta_{2} \mathrm{~m}$ loss represents a mechanism of immune escape, since this molecule could be lost as a result of increasing derangement in the transformed genome and a mutation "hot spot" at the $\beta_{2} m$ locus (46). Indeed, the mutability of this locus may have unexplored protective functions. Clearly, similar arguments can be advanced with regard to other events that decrease or eliminate MHC class I expression on the surface, such as loss of the MHC class I heavy chain or of transporters associated with antigen processing or low-molecular-weight proteins complex components.

To take another example, tumor cells (along with most normal cells) generally lack costimulatory molecules, such as B7-1 (CD80) and B7-2 (CD86), which are expressed on professional antigen-presenting cells and on a variety of other tissues after exposure to inflammatory cytokines (47). In the absence of costimulation, $T$ cells tend to become anergic. In the non-tumor-bearing setting, the absence of $\mathrm{B} 7$ molecule expression has been hypothesized to protect normal cells against autoreactivity. Does this finding help explain tumor cell escape from immune recognition? Transfection of tumor cells with both isoforms has been used successfully to trigger their immune-mediated rejection of experimental mouse tumors, which have some inherent immunogenicity. However, rejection is not observed when B7 molecules are inserted into less immunogenic tumors (48). Nonimmunogenicity is a category into which most, if not all, human tumors would fall; thus a lack of expression of the CD80 and CD86 costimulatory molecules is unlikely to be a global explanation for immune escape. Nonetheless, a greater understanding of the interactions of costimulatory molecules with negative regulatory molecules, such as cytotoxic $T$ lymphocyte-associated antigen 4 (CTLA-4), may enable more directed interventions (4). 


\section{Controlled unresponsiveness and negative regulation of antitumor $T$ cells}

Low-affinity autoreactive $\mathrm{T}$ cells can avoid negative selection in the thymus. Indeed a low level of autoreactivity is required for positive thymic selection. In normal circumstances, after maturation is complete, these autoreactive $\mathrm{T}$ cells are likely to be either ignorant (that is, they simply do not "see" their target epitope) or anergic (defined as a state of induced unresponsiveness). In the first case, they do not have any contact with the antigen that alters their phenotype or function. In the latter case, they are negatively regulated by host factors, such as cytokines, accessory molecules on antigen-presenting cells, and suppressor cells (49). Although there is undoubtedly some degree of ignorance to tumor antigens, there is clear evidence that tumor cells sensitize host $\mathrm{T}$ cells to tumor antigens (our unpublished data).

The spontaneous activation of host tumor-specific $\mathrm{T}$ cells is rarely sufficient to lead to tumor eradication. Interestingly, a very recent study has revealed a molecular mechanism mediated by a negative regulatory protein, Tob, a member of an antiproliferative gene family, in anergized $T$ cells (50). These data may point the way to a new understanding of how cells maintain unresponsiveness to antigen.

Other candidate negative regulatory mechanisms that may keep an incipient antitumor response in check include active negative regulatory mechanisms mediated by $\mathrm{CD} 4{ }^{+} \mathrm{CD} 25^{+}$suppressor cells, IL-13-secreting NKT cells, and $\mathrm{CD} 11 \mathrm{~b}^{+} \mathrm{Gr}-1^{+}$suppressor cells. The $\mathrm{CD} 4^{+} \mathrm{CD} 25^{+} \mathrm{T}$ cell population was first found to inhibit proliferation of $\mathrm{CD} 4^{+} \mathrm{CD} 25^{-} \mathrm{T}$ cells ex vivo as a result of TCR ligation and IL-2 activation. Extensive studies of suppressor/regulatory $\mathrm{T}$ cells in mouse models have demonstrated their importance in inhibition of autoimmunity (51). $\mathrm{CD}^{+} \mathrm{CD} 25^{+}$suppressor $\mathrm{T}$ cells also exist in humans and can inhibit proliferation and cytokine release in $\mathrm{CD} 4^{+} \mathrm{CD} 25^{-} \mathrm{T}$ cells by unknown mechanisms (52). IL-13 production following CD1 molecule ligation on NKT cells may also limit antitumor responses. In tumor-bearing mice, CD1 is upregulated on NKT cells. Knocking out CD1 promotes tumor resistance in mice. Similar results were also seen in anti-IL-13-treated mice (53). We do not yet know the relevance of this type of negative regulation to the immunotherapy of human cancer. Finally, like $\mathrm{CD} 4^{+} \mathrm{CD} 25^{+} \mathrm{T}$ cells and NKT cells, CD $11 \mathrm{~b}^{+} \mathrm{Gr}-1^{+}$ myeloid cells may send negative regulatory signals to $\mathrm{T}$ cells, triggering apoptotic death in $\mathrm{CD}^{+} \mathrm{T}$ cells following vaccination with powerful immunogens (54). Surprisingly, many mouse and human tumors produce GM-CSF, which can stimulate highly inhibitory $\mathrm{CD} 11 \mathrm{~b}^{+} \mathrm{Gr}-1^{+}$cells (55). The manipulation of each of these types of negative regulatory cells could be useful in the treatment of autoimmune disease and cancer.

\section{Future directions: a focus on $\mathrm{T}$ cell activation and death}

Significant evidence indicates that the central reasons for the failed antitumor immune response may be deficiencies in the maintenance of sustained tumor-specif- ic $\mathrm{T}$ cell activation. It is now clear that there are many ways in which triggering a TCR can result in the ultimate inactivation or even demise of the $T$ cell bearing it. The difference between antigen presentation in the tumor environment and that in a virally infected tissue is likely the activation of resident antigen-presenting cells, the scavengers and "danger" sensors of the immune system. The lack of proinflammatory mediators that induce maturation of dendritic cells, in conjunction with the abundant antigen presentation by non-costimulatory, tolerizing tumor cells, is the factor that may tip the $\mathrm{T}$ cell activation-inactivation balance in favor of tumor-specific $T$ cell tolerance. On the other hand, overstimulation can terminate an otherwise effective $T$ cell response through activation-induced cell death, fratricide, or exhaustion (56).

Although new antigen discovery and epitope mapping continue to be an important part of tumor immunology, few would dispute that several excellent targets expressed on a range of tumor histologies are now available. The next important breakthrough in cancer immunotherapy may come from an understanding of how to enhance $T$ cell avidity, how to maintain $\mathrm{T}$ cell activation while preventing $\mathrm{T}$ cell apoptosis, and how to reduce or eliminate the effects of negative regulatory factors.

In animal models, a number of new transgenic mouse models are now available, allowing for a reductionistic study of tumor interactions with elements of the innate and adaptive immune system. One particularly fruitful area currently under development involves the use of TCR transgenic mice. It is now clear that very large numbers of tumor-specific transgenic $\mathrm{CD}^{+}$and $\mathrm{CD}^{+}$ $T$ cells have little effect on the growth rate or lethality of syngeneic tumor cells that express the antigens targeted by these transgenic $T$ cells. These transgenic mouse systems model key aspects of increased tumor-specific $T$ cells found in some patients with cancer after active immunization. Using these models, we and others are evaluating cellular and molecular mechanisms in T cell activation, death, and anergy as they relate to the development of more effective cancer vaccines.

\section{Conclusions}

Tumor immunologists have made great strides in understanding the components of the successful immunotherapy of cancer. We have cloned antigens that are expressed by tumors, processed and presented in the context of MHC class I and class II molecules, and recognized by cells from the patient's own $\mathrm{T}$ cell repertoire. We have also learned how to immunize and are now capable of significantly expanding precursor $T$ cells with vaccination. Still, the proper and continued activation of antitumor $T$ cells remains a crucial missing piece of the immunotherapy puzzle and a significant barrier to developing an effective therapeutic vaccine. Thus, the focus of tumor immunotherapy is shifting. The challenge now is to learn how to promote $\mathrm{T}$ cell activation and proliferation while abrogating $\mathrm{T}$ cell anergy and death in the context of a profoundly tolerogenic tumor environment. 
1. Rosenberg, S.A., et al. 1985. Observations on the systemic administration of autologous lymphokine-activated killer cells and recombinant interleukin-2 to patients with metastatic cancer. N. Engl. J. Med 313:1485-1492.

2. Albert, M.L., et al. 1998. Tumor-specific killer cells in paraneoplastic cerebellar degeneration. Nat. Med. 4:1321-1324.

3. Overwijk, W.W., et al. 1999. Vaccination with a recombinant vaccinia virus encoding a "self" antigen induces autoimmune vitiligo and tumor cell destruction in mice: requirement for CD4(+) T lymphocytes. Proc. Natl. Acad. Sci. USA. 96:2982-2987.

4. van Elsas, A., Hurwitz, A.A., and Allison, J.P. 1999. Combination immunotherapy of B16 melanoma using anticytotoxic T lymphocyteassociated antigen 4 (CTLA-4) and granulocyte/macrophage colonystimulating factor (GM-CSF)-producing vaccines induces rejection of subcutaneous and metastatic tumors accompanied by autoimmune depigmentation. J. Exp. Med. 190:355-366.

5. Cox, A.L., et al. 1994. Identification of a peptide recognized by five melanoma-specific human cytotoxic T cell lines. Science. 264:716-719.

6. Meunier, L., et al. 1996. Quantification of CD1a, HLA-DR, and HLA class I expression on viable human Langerhans cells and keratinocytes. Cytometry. 26:260-264.

7. Parkhurst, M.R., et al. 1996. Improved induction of melanoma-reactive CTL with peptides from the melanoma antigen gp100 modified at HLAA*0201-binding residues. J. Immunol. 157:2539-2548.

8. Tangri, S., et al. 2001. Structural features of peptide analogs of human histocompatibility leukocyte antigen class I epitopes that are more potent and immunogenic than wild-type peptide. J. Exp. Med. 194:833-846.

9. Slansky, J.E., et al. 2000. Enhanced antigen-specific antitumor immunity with altered peptide ligands that stabilize the MHC-peptide-TCR complex. Immunity. 13:529-538.

10. Lyons, D.S., et al. 1996. A TCR binds to antagonist ligands with lower affinities and faster dissociation rates than to agonists. Immunity. 5:53-61

11. Overwijk, W.W., et al. 1998. gp100/pmel 17 is a murine tumor rejection antigen: induction of "self"-reactive, tumoricidal T cells using highaffinity, altered peptide ligand. J. Exp. Med. 188:277-286.

12. Rosenberg, S.A., et al. 1998. Immunologic and therapeutic evaluation of a synthetic peptide vaccine for the treatment of patients with metastatic melanoma. Nat. Med. 4:321-327.

13. Greenberg, P.D., Kern, D.E., and Cheever, M.A. 1985. Therapy of disseminated murine leukemia with cyclophosphamide and immune Lyt$1+, 2$ - T cells. Tumor eradication does not require participation of cytotoxic T cells. J. Exp. Med. 161:1122-1134.

14. Surman, D.R., Dudley, M.E., Overwijk, W.W., and Restifo, N.P. 2000 Cutting edge: CD4+ T cell control of CD8+ T cell reactivity to a model tumor antigen. J. Immunol. 164:562-565.

15. Topalian, S.L., et al. 1994. Human CD4+ T cells specifically recognize a shared melanoma-associated antigen encoded by the tyrosinase gene. Proc. Natl. Acad. Sci. USA. 91:9461-9465.

16. Rosenberg, S.A., et al. 1990. Gene transfer into humans: immunotherapy of patients with advanced melanoma, using tumor-infiltrating lymphocytes modified by retroviral gene transduction. N. Engl. J. Med. 323:570-578.

17. Kim, S., et al. 2000. In vivo natural killer cell activities revealed by natural killer cell-deficient mice. Proc. Natl. Acad. Sci. USA. 97:2731-2736.

18. Diefenbach, A., Jensen, E.R., Jamieson, A.M., and Raulet, D.H. 2001. Rae1 and $\mathrm{H} 60$ ligands of the NKG2D receptor stimulate tumour immunity. Nature. 413:165-171.

19. Ruggeri, L., et al. 2002. Effectiveness of donor natural killer cell alloreactivity in mismatched hematopoietic transplants. Science. 295:2097-2100.

20. Restifo, N.P. 2001. Vaccines to die for. Nat. Biotechnol. 19:527-528.

21. Steinman, R.M., and Pope, M. 2002. Exploiting dendritic cells to improve vaccine efficacy. J. Clin. Invest. 109:1519-1526. doi:10.1172/JCI200215962.

22. Ying, H., et al. 1999. Cancer therapy using a self-replicating RNA vaccine. Nat. Med. 5:823-827.

23. Leitner, W.W., et al. 2000. Enhancement of tumor-specific immune response with plasmid DNA replicon vectors. Cancer Res. 60:51-55.

24. Palese, P., Zavala, F., Muster, T., Nussenzweig, R.S., and Garcia-Sastre, A. 1997. Development of novel influenza virus vaccines and vectors. J. Infect. Dis. 176(Suppl. 1):S45-S49.

25. Restifo, N.P. 1996. The new vaccines: building viruses that elicit antitumor immunity. Curr. Opin. Immunol. 8:658-663.

26. Rosenberg, S.A., et al. 1998. Immunizing patients with metastatic melanoma using recombinant adenoviruses encoding MART-1 or gp100 melanoma antigens. J. Natl. Cancer Inst. 90:1894-1900.

27. Letvin, N.L. 2002. Strategies for an HIV vaccine. J. Clin. Invest. 110:15-20. doi:10.1172/JCI200215985

28. Kedl, R.M., et al. 2000. T cells compete for access to antigen-bearing antigen-presenting cells. J. Exp. Med. 192:1105-1113.
29. Chen, W., Anton, L.C., Bennink, J.R., and Yewdell, J.W. 2000. Dissecting the multifactorial causes of immunodominance in class I-restricted $\mathrm{T}$ cell responses to viruses. Immunity. 12:83-93.

30. Stoler, D.L., et al. 1999. The onset and extent of genomic instability in sporadic colorectal tumor progression. Proc. Natl. Acad. Sci. USA. 96:15121-15126

31. Staveley-O'Carroll, K., et al. 1998. Induction of antigen-specific T cell anergy: an early event in the course of tumor progression. Proc. Natl. Acad. Sci. USA. 95:1178-1183.

32. Wang, M., et al. 1995. Active immunotherapy of cancer with a nonreplicating recombinant fowlpox virus encoding a model tumor-associated antigen. J. Immunol. 154:4685-4692.

33. McCabe, B.J., et al. 1995. Minimal determinant expressed by a recombinant vaccinia virus elicits therapeutic antitumor cytolytic $T$ lymphocyte responses. Cancer Res. 55:1741-1747.

34. D’Urso, C.M., et al. 1991. Lack of HLA class I antigen expression by cultured melanoma cells FO-1 due to a defect in B2m gene expression. J. Clin. Invest. 87:284-292.

35. Maio, M., Altomonte, M., Tatake, R., Zeff, R.A., and Ferrone, S. 1991. Reduction in susceptibility to natural killer cell-mediated lysis of human FO-1 melanoma cells after induction of HLA class I antigen expression by transfection with B2m gene. J. Clin. Invest. 88:282-289.

36. Restifo, N.P., et al. 1993. Molecular mechanisms used by tumors to escape immune recognition: immunogenetherapy and the cell biology of major histocompatibility complex class I. J. Immunother. 14:182-190.

37. Restifo, N.P., et al. 1993. Identification of human cancers deficient in antigen processing. J. Exp. Med. 177:265-272.

38. Restifo, N.P., et al. 1996. Loss of functional beta 2-microglobulin in metastatic melanomas from five patients receiving immunotherapy. J. Natl. Cancer Inst. 88:100-108.

39. Restifo, N.P. 2001. Countering the 'counterattack' hypothesis. Nat. Med. 7:259.

40. Restifo, N.P. 2000. Not so Fas: re-evaluating the mechanisms of immune privilege and tumor escape. Nat. Med. 6:493-495.

41. Restifo, N.P. 2000. Building better vaccines: how apoptotic cell death can induce inflammation and activate innate and adaptive immunity. Curr. Opin. Immunol. 12:597-603.

42. Chappell, D.B., Zaks, T.Z., Rosenberg, S.A., and Restifo, N.P. 1999 Human melanoma cells do not express Fas (Apo-1/CD95) ligand. Can cer Res. 59:59-62.

43. Kambayashi, T., et al. 2001. Purified MHC class I molecules inhibit activated NK cells in a cell-free system in vitro. Eur. J. Immunol. 31:869-875.

44. Smyth, M.J., and Snook, M.B. 1999. Perforin-dependent cytolytic responses in beta2-microglobulin-deficient mice. Cell. Immunol. 196:51-59.

45. Porgador, A., Mandelboim, O., Restifo, N.P., and Strominger, J.L. 1997. Natural killer cell lines kill autologous beta2-microglobulin-deficient melanoma cells: implications for cancer immunotherapy. Proc. Natl. Acad. Sci. USA. 94:13140-13145.

46. Parnes, J.R., Sizer, K.C., Seidman, J.G., Stallings, V., and Hyman, R. 1986. A mutational hot-spot within an intron of the mouse beta 2-microglobulin gene. EMBO J. 5:103-111.

47. Anderson, D.E., Sharpe, A.H., and Hafler, D.A. 1999. The B7CD28/CTLA-4 costimulatory pathways in autoimmune disease of the central nervous system. Curr. Opin. Immunol. 11:677-683.

48. Chen, L., et al. 1994. Tumor immunogenicity determines the effect of B7 costimulation on $\mathrm{T}$ cell-mediated tumor immunity. J. Exp. Med. 179:523-532

49. Antony, P.A., and Restifo, N.P. 2002. Do CD4+ CD25+ immunoregulatory T cells hinder tumor immunotherapy? J. Immunother. 25:202-206.

50. Tzachanis, D., et al. 2001. Tob is a negative regulator of activation that is expressed in anergic and quiescent T cells. Nat. Immunol. 2:1174-1182.

51. Shevach, E.M. 2000. Regulatory T cells in autoimmmunity. Annu. Rev. Immunol. 18:423-449.

52. Dieckmann, D., Plottner, H., Berchtold, S., Berger, T., and Schuler, G. 2001. Ex vivo isolation and characterization of CD4(+)CD25(+) T cells with regulatory properties from human blood. J. Exp. Med. 193:1303-1310.

53. Terabe, M., et al. 2000. NKT cell-mediated repression of tumor immunosurveillance by IL-13 and the IL-4R-STAT6 pathway. Nat. Immunol. 1:515-520.

54. Bronte, V., et al. 1998. Apoptotic death of CD8+ T lymphocytes after immunization: induction of a suppressive population of Mac-1+/Gr-1+ cells. J. Immunol. 161:5313-5320.

55. Bronte, V., et al. 1999. Unopposed production of granulocytemacrophage colony-stimulating factor by tumors inhibits CD8+ T cell responses by dysregulating antigen-presenting cell maturation. J. Immunol. 162:5728-5737.

56. Overwijk, W.W., and Restifo, N.P. 2001. Creating therapeutic cancer vaccines: notes from the battlefield. Trends Immunol. 22:5-7. 